\title{
Projection and Quantifier Elimination Using Non-uniform Cylindrical Algebraic Decomposition
}

\author{
Christopher W. Brown \\ United States Naval Academy \\ Annapolis, Maryland 21402 \\ wcbrown@usna.edu
}

\begin{abstract}
Cylindrical Algebraic Decomposition (CAD) is an established tool in the computer algebra community for computing with semialgebraic sets / Tarski formulas. The key property of CAD is that it provides a representation in which geometric projection and set complement (the analogues of the logical operations of quantifier elimination and negation for Tarski formulas) are trivial. However, constructing a CAD often requires an impractical amount of time and space. Non-uniform $\mathrm{CAD}(\mathrm{NuCAD})$ was introduced with the goal of providing a more practically efficient alternative to $\mathrm{CAD}$ for computing with semi-algebraic sets / Tarski formulas. As a first step towards that goal, previous work has shown that Open NuCADs do provide a much more efficient representation than Open CADs. However, it hasn't been shown that the key operation of projection can be computed efficiently in the NuCAD representation, because while set complement is trivial for NuCADs, as it is for CADs, projection, in contrast to the $\mathrm{CAD}$ case, is not. This paper provides another step towards the larger goal by showing how projection can be done efficiently in the Open NuCAD representation.

The importance of this contribution is not restricted to Open $\mathrm{NuCADs}$, since the same approach to projection will carry over to the general case for NuCADs where, we hope, the practical benefits of the much smaller representation NuCAD provides will be even greater.
\end{abstract}

\section{CCS CONCEPTS}

- Mathematics of computing $\rightarrow$ Solvers;

\section{KEYWORDS}

cylindrical algebraic decomposition; polynomial inequalities; real constraint solving

\section{ACM Reference format:}

Christopher W. Brown. 2017. Projection and Quantifier Elimination Using Non-uniform Cylindrical Algebraic Decomposition. In Proceedings of ISSAC '17, Kaiserslautern, Germany, fuly 25-28, 2017, 8 pages.

https://doi.org/http://dx.doi.org/10.1145/3087604.3087651

This work supported by NSF grant 1525896 .

This paper is authored by an employee(s) of the United States Government and is in the public domain. Non-exclusive copying or redistribution is allowed, provided that the article citation is given and the authors and agency are clearly identified as its source.

ISSAC '17, July 25-28, 2017, Kaiserslautern, Germany

2017. ACM ISBN ISBN 978-1-4503-5064-8/17/07 ..\$15.00

https://doi.org/http://dx.doi.org/10.1145/3087604.3087651

\section{INTRODUCTION}

Tarski formulas and semi-algebraic sets are fundamental objects of study in computer algebra. ${ }^{1}$ Cylindrical Algebraic Decomposition $(\mathrm{CAD})$ is an established tool for computing with semi-algebraic sets and Tarski formulas. CAD provides an explicit representation of semi-algebraic sets that can be constructed from Tarski formulas, which are implicit representations. The CAD representation supports many operations, but the most important are projection and set complement, which allow CAD to be used for quantifier elimination. Specifically, if $F$ is a quantifier-free formula in variables $x_{1}, \ldots, x_{n}$, then a CAD representation of $Q_{r+1} x_{r+1} \cdots Q_{n} x_{n} F$, where the $Q_{i}$ are $\forall$ or $\exists$, can be constructed by first constructing a CAD of $\mathbb{R}^{n}$ representing the set defined by $F$, and second performing a combination of projections and set complements to arrive at a $\mathrm{CAD}$ of $\mathbb{R}^{r}$ representing the set defined by the quantified formula.

It is well-known that constructing a CAD from a Tarski formula in $n$ variables has doubly-exponential worst-case running time and space [2]. In fact, even just the number of 1-level cells constructed is, in the worst case, doubly exponential in the number of variables. Moreover, practical experience has shown that the time and space inefficiencies of CAD often make even moderate sized problems impractical. Non-uniform CAD (NuCAD) was introduced with the goal of providing a more efficient alternative to $\mathrm{CAD}$ [1], using ideas from Jovanovic \& de Moura's NLSat [3] (see [6] for an alternative approach to trying to leverage those ideas in CAD construction). Ultimately, meeting that goal requires not only that a NuCAD constructed from a given quantifier-free formula is more efficient than a CAD constructed from that formula, but also that NuCAD efficiently supports those crucial operations that CAD supports: most crucially, projection and set-complement. Since set-complement is trivial in both representations (simply swap labels on cells), the key issue is how to do projection efficiently for NuCADs, and that is what this paper addresses.

An "Open" CAD is a weak form of CAD that consists only of full-dimensional cells. It's not a true decomposition of $\mathbb{R}^{n}$, because there are points that are not in any cells. However, such points comprise a lower-dimensional subset of $\mathbb{R}^{n}$. Open CAD has appeared in, for example, $[4,5]$ where it is noted that projection and lifting are conceptually simpler, and faster than for the general case. For certain problems, e.g. satisfiability of strict inequalities and determining semi-definiteness, Open CAD suffices. What [1] actually defines is Open NuCAD, and an algorithm for constructing them from Tarski formulas. In this paper, we continue to work with Open

\footnotetext{
${ }^{1}$ A Tarski formula is a boolean combination of sign conditions on polynomials, possibly with quantifiers. A semi-algebraic set is a subset of $\mathbb{R}^{n}$ for which there is a quantifierfree Tarski formula $F$ in $x_{1}, \ldots, x_{n}$ such that $\alpha \in \mathbb{R}^{n}$ is in the set precisely when $F(\alpha)$ is true.
} 


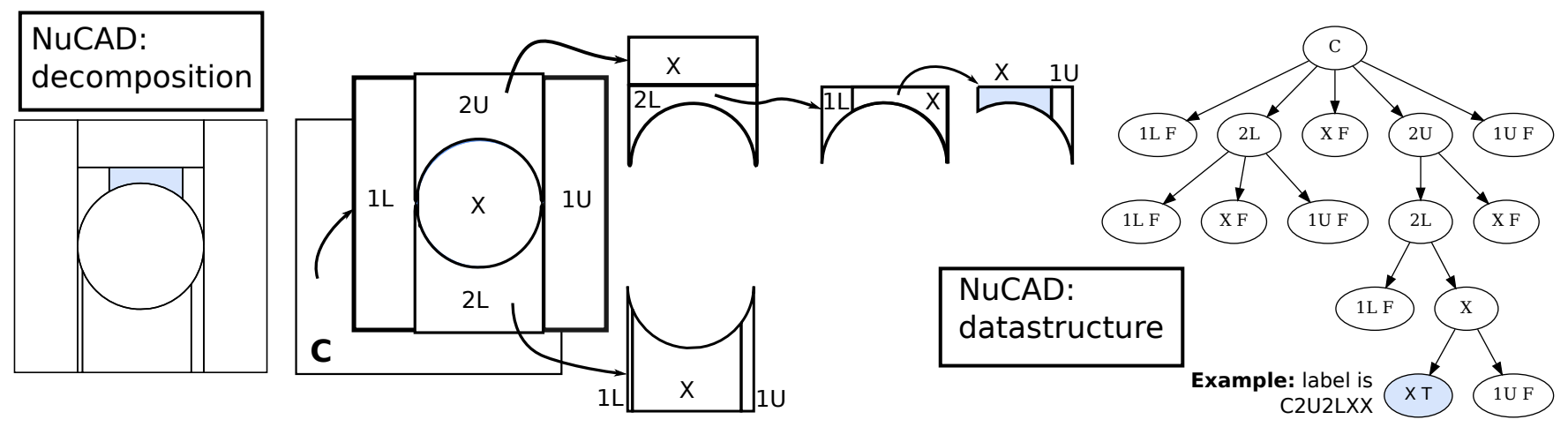

Figure 1: This figure shows the correspondence between NuCAD as a hierarchical decomposition of $\mathbb{R}^{n}$ and NuCAD as a datastructure. Note that leaf cells have T/F (TRUE/FALSE) labels. The semi-algebraic set defined by a NuCAD is the union of the TRUE cells. Relative to its parent, each cell is labeled with either $X, k L$ or $k U$. Globally, each cell is uniquely labeled by the concatenation of these relative labels along the path from the root node to the cell. Thus the highlighted cell has label $C 2 U 2 L X X$.

NuCAD because the initial development is simpler, and if there are efficiency gains in the "open" case, the gains to be had in the general case are likely to be still greater. The purpose of this paper is, in the context of Open CAD and NuCAD, to: 1) introduce an algorithm for computing projections of semi-algebraic sets in the NuCAD representation, 2) present experimental results demonstrating that the time required to compute the projection of a semi-algebraic set $S$ from its NuCAD representation is small relative to the time required to construct the $\mathrm{NuCAD}$ for $S$ in the first place, 3) present experimental results demonstrating that the number of cells in the result of computing the projection of semi-algebraic set $S$ in the $\mathrm{NuCAD}$ representation is small compared to the number of cells in the result of computing the projection of $S$ using partial CAD, and 4) present experimental results demonstrating that as problems get larger there are projections that can be computed using NuCAD that cannot be computed (in practice) with CAD.

\section{BACKGROUND}

We assume the reader is already familiar with the usual CAD notions - like delineability, level of a polynomial, etc. Note that $\pi_{k}(\cdot)$ denotes projection down onto $\mathbb{R}^{k}$. This paper deals with open cylindrical cells which, except in the trivial case of a single cell, cannot truly decompose $\mathbb{R}^{n}$. Instead, we say that a set of open regions defines a weak decomposition of $\mathbb{R}^{n}$ if the regions are pairwise disjoint, and the union of their closures contains $\mathbb{R}^{n}$. Open CADs and Open $\mathrm{NuCADs}$ are weak decompositions of real space. By labeling cells as TRUE or FALSE, these decompositions provide representations of semi-algebraic sets that are correct up to lower-dimensional components. Specifically, all cells are correctly marked as completely in or out of the semi-algebraic set, but the boundaries between cells (which are of lower dimension) are not part of the decomposition, so whether we consider boundaries to be in the set or out, we may be miscategorizing some points in lower-dimensional components.

In this paper we would like to represent the projection of a semialgebraic set as an Open CAD or NuCAD. To this end we define the notion of a weak projection, and we will show in this paper how to construct a NuCAD of $r$-space that is a weak projection of the semi-algebraic set defined by a NuCAD of $n$-space.
Definition 2.1. If $S$ is an open semi-algebraic subset of $\mathbb{R}^{n}$, and $r \leq n$ a positive integer, we say that an open set $W \subseteq \mathbb{R}^{r}$ is a weak projection of $S$ onto $\mathbb{R}^{r}$ if $W \subseteq \pi_{r}(S)$ and there are no open subsets of $\pi_{r}(S)-W$.

It's worth noting that in this paper, the difference between the true projection and the weak projection onto $r$-space we compute using NuCADs will be a subset of some variety defined by a nonzero polynomial in $r$ variables.

Here we recall the basic definitions of Open NuCAD (see [1]), which are also illustrated in Figure 1.

Definition 2.2. An Open Cylindrical Cell is a subset of $\mathbb{R}^{n}$ of the form $\left\{\left(\alpha_{1}, \ldots, \alpha_{n}\right) \in B \times \mathbb{R} \mid f\left(\alpha_{1}, \ldots, \alpha_{n-1}\right)\right.$

$\left.<\alpha_{n}<g\left(\alpha_{1}, \ldots, \alpha_{n-1}\right)\right\}$ or $\left\{\left(\alpha_{1}, \ldots, \alpha_{n}\right) \in B \times \mathbb{R} \mid f\left(\alpha_{1}\right.\right.$,

$\left.\left.\ldots, \alpha_{n-1}\right)<\alpha_{n}\right\}$ or $\left\{\left(\alpha_{1}, \ldots, \alpha_{n}\right) \in B \times \mathbb{R} \mid \alpha_{n}<g\left(\alpha_{1}\right.\right.$,

$\left.\left.\ldots, \alpha_{n-1}\right)\right\}$ where $B$ is an open cylindrical cell in $\mathbb{R}^{n-1}$ and the graphs of $f$ and $g$ over $B$ are disjoint sections of polynomials, and () is considered an open cylindrical cell in $\mathbb{R}^{0}$.

As a data structure, an Open NuCAD Cell represents a cylindrical cell as an array $C$ of bounds descriptions: so $C[i]$ is a description of the cell's upper and lower bounds for $x_{i}$. Additionally, a cell data structure stores a sample point from inside the cell, and potentially carries a truth label, a label describing its position within a NuCAD data structure, and a list of child cells within a NuCAD data structure.

Definition 2.3. An Open Non-uniform Cylindrical Algebraic Decomposition (Open NuCAD) of $\mathbb{R}^{n}$ is a collection $C$ of open cylindrical cells, each of which is labeled with a unique string of the form $C(X \mid[0-9]+(L \mid U)) *$. The relation $E=\left\{\left(C_{1}, C_{2}\right) \mid C_{1}, C_{2}\right.$ are cells with labels $l a b_{1}$ and $l a b_{2}$ satisfying $\left.l a b_{2}=l a b_{1}(X \mid[0-9]+(L \mid U))\right\}$ defines a graph on the cells such that

(1) the graph $(C, E)$ is a tree, rooted at the cell consisting of all of $\mathbb{R}^{n}$, which is labeled $C$,

(2) the children of cell $C_{0}$ with label $l a b_{0}$ have labels from taken from the set $\left\{l a b_{0} 1 L, \ldots, l a b_{0} n L, l a b_{0} 1 U, \ldots, l a b_{0} n U, l a b_{0} X\right\}$ and if $C_{0}$ has children, then one of them is labeled $l a b_{0} X$,

(3) if cell $C_{2}$ is the child of $C_{1}$ with label $l a b_{1} X$, then $C_{2} \subset C_{1}$ and for each $i \in\{1, \ldots, n\}$, in the cylinder over $\pi_{i-1}\left(C_{2}\right)$ the 
section that defines the lower (resp. upper) boundary of $C_{2}$ in $x_{i}$ is either identical to or disjoint from the section that defines the lower (resp. upper) boundary of $C_{1}$ in $x_{i}$

(4) if cell $C_{X}$ is the child of $C_{0}$ with label $l a b_{0} X$, then for all $i \in$ $\{1, \ldots, n\}$

$$
\left(\pi_{i-1}\left(C_{X}\right) \times \mathbb{R}\right) \cap \pi_{i}\left(C_{0}\right)-\pi_{i}\left(C_{X}\right)
$$

consists of zero, one or two open cells: the region with $x_{i}$ coordinates below $\pi_{i}\left(C_{X}\right)$ if it is non-empty, which is denoted $B_{L}$, and the region with $i$-coordinates above $\pi_{i}\left(C_{X}\right)$ if it is non-empty, which is denoted $B_{U}$. There is a cell with label $l a b_{0} i L$ if and only if $B_{L}$ is non-empty and, if it exists, that cell is $\left(B_{L} \times \mathbb{R}^{n-i}\right) \cap C_{0}$. There is a cell with label $l a b_{0} i U$ if and only if $B_{U}$ is non-empty and, if it exists, it is $\left(B_{U} \times \mathbb{R}^{n-i}\right) \cap C_{0}$.

We note that in point 3 of the definition, the requirement that $C_{2}$ is a strict subset of $C_{1}$ is a change from the definition given in [1]. This means that a cell is either a leaf, or it has multiple children. There are several operations we will assume are defined for an Open Cylindrical Cell $C$ within a NuCAD data structure:

- getSamplePoint() : returns the sample point of $C$.

- $\operatorname{get} C h i l d(\omega)$ : returns the child with label $\omega$ relative to $C$, or FAIL if no child has that label.

- getXLevel() : returns the split-level (see Definition 3.1) of the $X$-child of $C$, or 0 if $C$ is not a leaf. Note that this is the maximum of the split-levels of $C$ 's children, and note that there must be an $L$-child or $U$-child with that split-level, in addition to the $X$-child.

- locateLeaf $(\alpha)$ : given point $\alpha \in \pi_{i}(C)$, returns a leaf cell $C^{\prime}$ from the subtree rooted at $C$ such that $\alpha \times \mathbb{R}^{n-i} \cap C^{\prime} \neq \emptyset$. If $\alpha$ lies on a boundary separating cells in the subtree rooted at $C$, "FAIL" plus some extra information is returned.

- moveSamplePoint $(\alpha)$ : given point $\alpha \in \pi_{i}(C)$, moves the sample point of $C$ to lie on $\alpha \times \mathbb{R}^{n-i}$.

We do not define locateLeaf and moveSamplePoint here, due to space considerations, but we note that they just involve a number of univariate real-root isolations for polynomials with rational coefficients. Additionally, there are two algorithms related to NuCAD construction that we need.

- $E \leftarrow$ OCMerge $\left(C_{1}, C_{2}, k\right)$ : given two cells whose sample points agree in the first $k$ coordinates, construct $E$, a subcell of $C_{1}$ such that $\pi_{k}(E) \subseteq \pi_{k}\left(C_{2}\right)$. There is not space here to describe this algorithm, though we remark that if $Q$ is the set of level 1 through $k$ boundary polynomials of $C_{2}$, the OC-Merge-Set algorithm, described in [1], applied to $C_{1}$ and $Q$ suffices to do this.

- $Q \leftarrow \operatorname{SplitByCell}\left(C_{1}, C_{2}, k\right)$ : given leaf cell $C_{1}$ and cell $C_{2} \subseteq C_{1}$, splits $C_{1}$ into subcells that become its children, such that what becomes the $X$-child of $C_{1}$ is $C_{1} \cap\left(\pi_{k}\left(C_{2}\right) \times \mathbb{R}^{n-k}\right)$. A queue of all the children added other than the $X$-child is returned as " $Q$ ". There is not enough space to define this algorithm, though we note that lines 15-35 of the algorithm "Split" from [1] provides one.

\section{TREE-BASED PROJECTION}

The notion of level is fundamental to descriptions of CAD. The CAD data structure consists of a tree in which each vertex is associated with a (cylindrical) cell, and the level of a cell is its depth in that tree. In that data structure, a $k$-level cell is a subset of $\mathbb{R}^{k}$, and

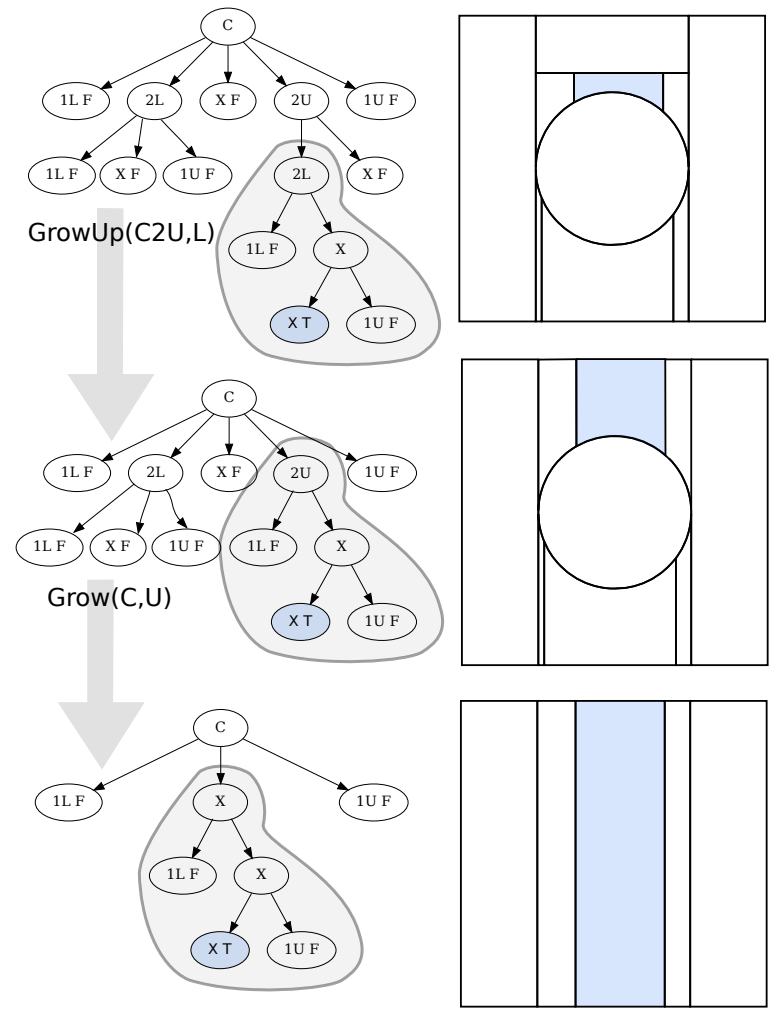

Figure 2: Projection based on the NuCAD tree.

the requirement that cells be cylindrically arranged means that the projection of a $k$-level cell onto $\mathbb{R}^{j}$, where $j<k$, is the cell's $j$-level ancestor in the tree. In a NuCAD, by contrast, each cell in the NuCAD tree is a subset of $\mathbb{R}^{n}$, so the notion of level from CAD does not carry over. We next provide a useful definition of level both for an individual cell, and for a subtree in the NuCAD tree.

Definition 3.1. Let $C$ be a cell in $D$, an Open NuCAD of $\mathbb{R}^{n}$. If $C^{\prime}$ is a cell in the subtree rooted at $C$, we say that the level within $C$ of $C^{\prime}$ is $i$ if $C^{\prime}$ is exactly $\left(S \times \mathbb{R}^{n-i}\right) \cap C$ for some $S \subseteq \mathbb{R}^{i}$. By extension, we say that the level of the subtree rooted at $C$ is $i$ if all cells in the subtree (excluding $C$ itself) have level at most $i$, and at least one has level exactly equal to $i$, or 0 if $C$ is a leaf. Finally, the split-level of cell $C$ is its level within its parent cell, or 0 for the root node.

Projection onto a lower dimensional space of a semi-algebraic set represented by a $\mathrm{CAD}$ is trivial (given that you already have a $\mathrm{CAD}$ !) because it can be done solely based on the CAD tree structure no algebraic computation required. While this is not the case for NuCADs, there are some projections that can be computed this way, i.e. solely with non-algebraic operations on the NuCAD tree. We refer to this as "easy projection", and describe it in this section.

The fundamental idea behind "easy projection" can be seen illustrated in Figure 2. In the top NuCAD tree, we see that cell $C 2 U$ has two children of split-level 2: a $2 L$-child and an $X$-child. The structure of NuCADs is such that these two sibling cells must have the same projection onto $\mathbb{R}^{1}$. Since the $x$-child has only FALSE cells in its subtree (in fact, it is the only cell in its subtree, being a leaf), we could "grow" the cells from the $2 L$-child upwards to cover the 
entire region of the $X$-child, thereby eliminating the $X$-child, and the projection of the subtree rooted at $C 2 U$ would be unaltered. Figure 2 shows this change in the transition from the top NuCAD to the middle NuCAD. Moreover, it shows that this change in the $\mathrm{NuCAD}$ decomposition is effected by a simple operation on the NuCAD tree. We call this a "GrowUp" operation.

The transition from the middle NuCAD to the bottom in Figure 2 illustrates the same idea. Cell $C$ has three children of splitlevel 2: $2 L, X$ and $2 U$. The subtrees rooted at the $2 L$ and $X$ children of $C$ contain only FALSE cells, so we can "grow" the $2 U$-child down to cover both the $2 L$ and $X$ children, and the projection onto 1-space of the NuCAD still defines the same set. Moreover, once again, this change is effected by a simple operation on the NuCAD tree.

These ideas lead to three algorithms, GrowUp, GrowDown and Grow, that expand one cell and its subtree to "absorb" its one or more of its siblings.

- GrowUp $(C, f)$ : grow $f$-child of $C$ to absorb the sibling directly "above" it.

- GrowDown $(C, f)$ : same as GrowUp, but in the other direction.

- Grow $(C, f)$ : grow $f$-child of $C$ to absorb both sibling cells of its split-level.

We give an explicit description of GrowUp as Algorithm 1. The others follow the same ideas. The following theorem regarding GrowUp is stated, though its proof is left out for space considerations.

Theorem 3.2. Let $C$ be a cell, $C_{X}$ its $X$-child, and $r$ the split-level of $C_{X}$. If $\operatorname{Grow} U p(C, L)$ is called, and either $C_{X}$ has every cell marked FALSE or the rL-child (call it $C_{L}$ ) has every cell within it marked TRUE, then for any $k<r$, the projection onto $\mathbb{R}^{k}$ of $C$ prior to the call of GrowUp is the same as it is after the call. Similarly, If GrowUp $(C, X)$ is called, and either $C_{X}$ has every cell marked TRUE or the $r U$-child (call it $C_{U}$ ) has every cell within it marked FALSE, then for any $k<r$, the projection onto $\mathbb{R}^{k}$ of C prior to the call of GrowUp is the same as it is after the call.

Finally, we define Algorithm 2, EasyProjection, which projects cells based only on the structure of the NuCAD graph. It is incomplete, in the sense that it is not necessarily able to project away all cells of level greater than the target level $k$. A precise characterization of when and why this happens appears later.

Theorem 3.3. Algorithm 2 meets its specifications.

Proof. We give a sketch of the key points of this proof here. First, note that the "FAIL" case (lines 1,2) is there to avoid having to constantly check whether cell $C$ has a $U$ or an $L$ child for a particular level. A quick look at how it is used within the algorithm should show you that such results are essentially ignored. Also note that when $C$ is a leaf, the value $(0, t)$ returned in $C$ is what the specification requires. This will allow us to proceed by induction on the number of nodes in the subtree rooted at $C$, since we have taken care of the base case here.

We next consider the case in which the initial value of $r$ from line 6 is less than or equal to $k$. In this case, by induction, $r_{X}$ is the level of the subtree rooted at $C$, and $t_{X}$ is TRUE (resp. FALSE) exactly when all cells in that subtree are TRUE (resp. FALSE), and UNDET otherwise. We will skip over the while-loop, and after lines 26-32 we will have $r_{\max }=r_{X}$ and $t=t_{X}$. Lines 33-38 will call

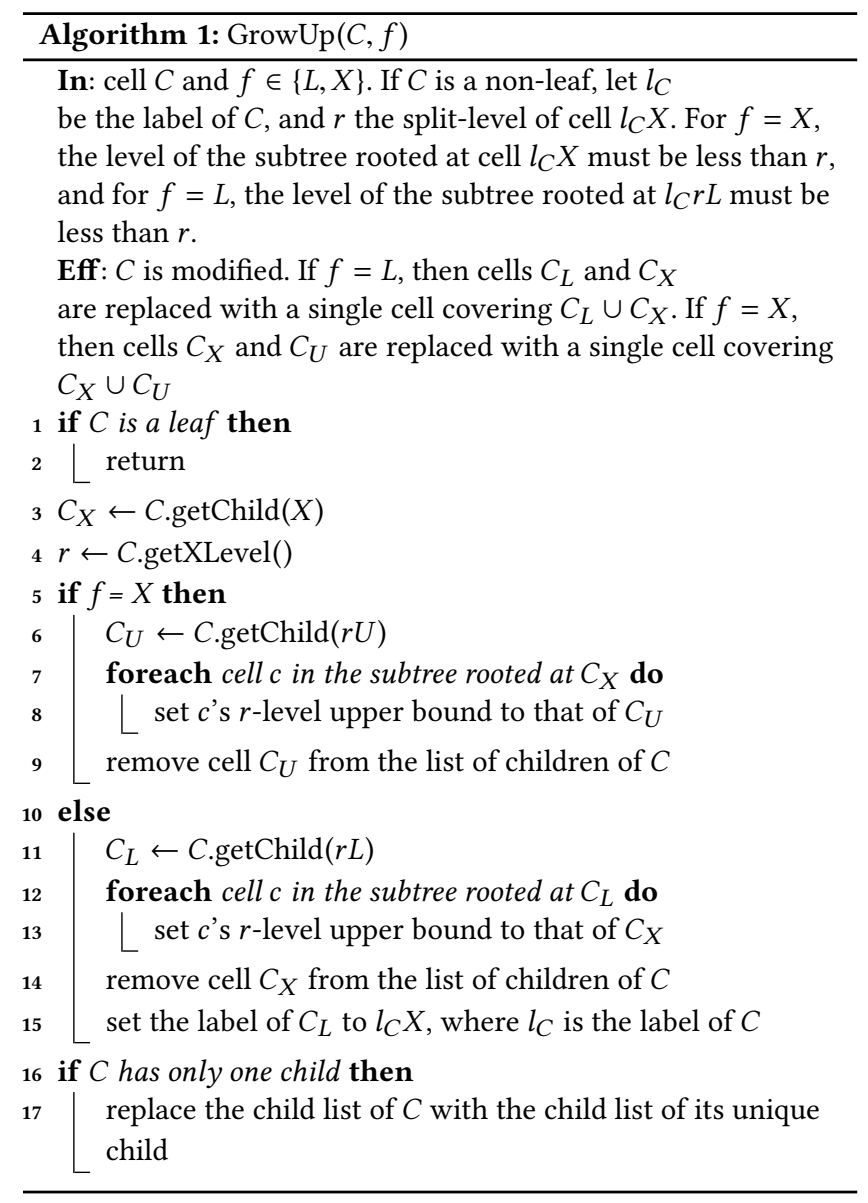

EasyProjection recursively on the other children of $C$, updating $r_{\max }$ on line 36 if a higher-level descendant cell is encountered, and setting $t=$ UNDET if anything other than the value of $t_{X}$ is encountered. Thus, on line 39 , the returned result $\left(r_{\max }, t\right)$ meets the specification.

Finally, we consider the general situation, where the initial splitlevel of $C_{X}$ is greater than $k$. Thus we actually enter the first while loop. We exit that loop in one of two distinct cases. The first case is $r=r_{\text {prev }}$ (which implies $r>k$ ). This happens when, after a pass through the loop body, the split-level of the $X$-child has not been reduced, which means there is an $L$ or $U$ child of that same split-level. What we require is that $r_{\max }$ is the maximum of the split-level of these cells and any cells in their subtrees, and $t$ is TRUE (resp.False) if all the leaves of the subtrees rooted at these cells are TRUE (resp.FALSE), and UNDET otherwise. If we have left the loop this way, the only children of $C$ on which EasyProjection has not been called have level less than $r$. The remaining lines, 31-39, simply call EasyProjection on those remaining children, updating $r_{\max }$ and $t$ appropriately.

The second case is when $r \neq r_{\text {prev }}$ but $r \leq k$. In this case, we only need $r_{\text {max }}$ to be the maximum of the split-level of $C_{X}$ and all the cells in its subtree, and $t$ to be TRUE (resp.FALSE) if all the leaves of only the subtree rooted at $C_{X}$ are TRUE (resp.FALSE), and UNDET otherwise. In this case, the remaining lines of the algorithm (i.e. 


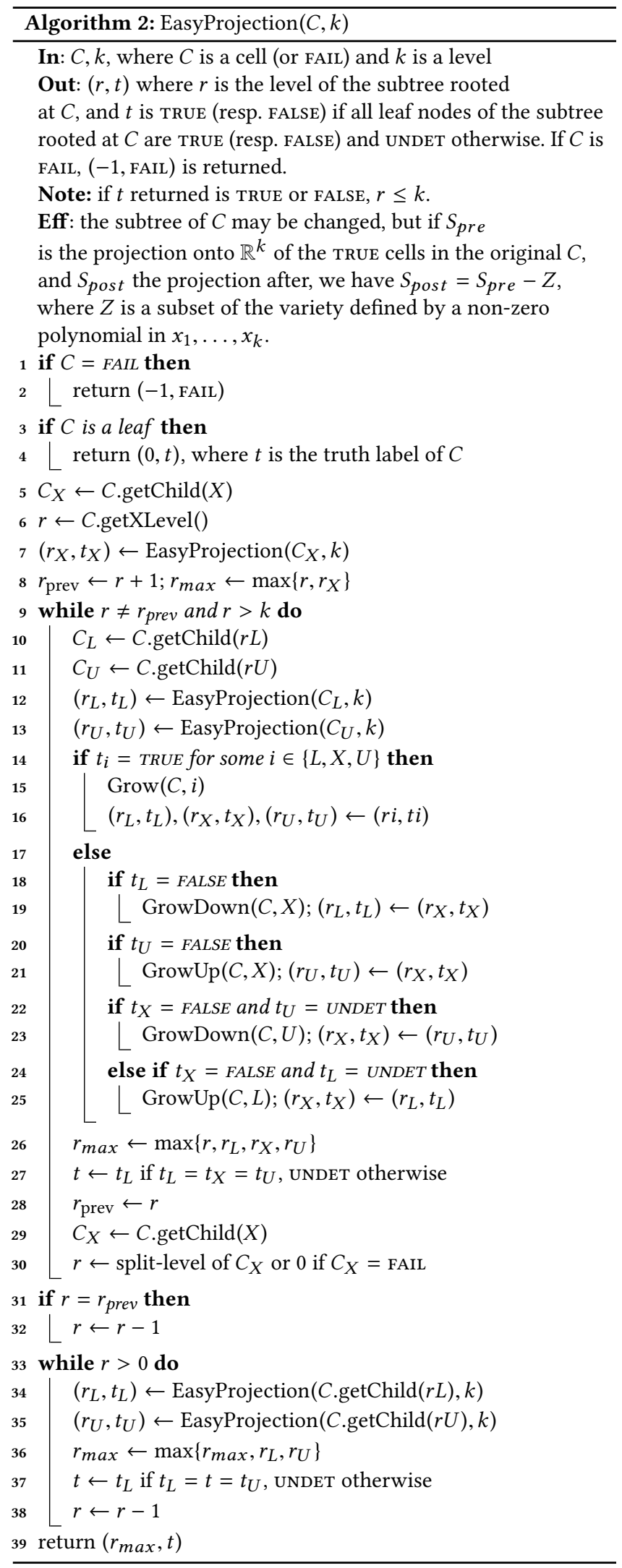

31-39) operate just as in the case in which the first while-loop was never entered.

At this point, the full proof of correctness would require verifying that the first while-loop exits satisfying the conditions required of these two cases. Due to space constraints, we do not include that argument. Conceptually it is not difficult, but its presentation is complicated by the proliferation of cases in which a $U$ or $L$ child of a given level may or may not exist.

EasyProjection $(C, k)$ may not completely project $C$ onto level $k$, meaning that there may be cells in the tree with split-level greater than $k$. The following theorem characterizes the NuCAD tree after such a call to Easy Project.

Theorem 3.4. If EasyProjection $(C, k)$ returns $(r, t)$ where $r>k$, then there exists a cell $C^{\prime}$ in the subtree rooted at $C$ (after the call to EasyProjection) with two distinct child cells of level $C^{\prime}$.getXLevel() that both have subtrees that a) are of level less than or equal to $k$, and b) contain both TRUE and FALSE cells.

Proof. We proceed by induction on the number of cells in the subtree rooted at $C$.

Case 1: after the call, the subtrees of all children of $C$ have level at most $k$, but $C$ has children of split-level greater than $k$. Let $C_{X}$ be the $X$-child of $C$ and, $C_{L}$ its sibling of the same level (for simplicity assume $C_{X}$ has no $U$-sibling). If either $C_{X}$ or $C_{L}$ has all true or all FALSE cells, then EasyProjection $(C, k)$ would have merged them in lines 14-25. So $C_{X}$ and $C_{L}$ must contain both TRUE and FALSE cells, and the theorem holds taking $C$ itself as $C^{\prime}$. This is the base case of our induction.

Case 2: after the call, there is a child of $C$ with a subtree of level greater than $k$. In this case, the subtree rooted at that child must contain both TRUE and FALSE cells. So there must have been a recursive call, EasyProjection $\left(C^{*}, k\right)$ that returned $\left(r^{*}, t^{*}\right)$ where $r^{*}>k$. In this case, induction says that the subtree rooted at $C^{*}$ has a cell $C^{\prime}$ with two distinct child cells of level $C^{\prime}$.getXLevel() that both have subtrees that are of level less than or equal to $k$, and contain both TRUE and FALSE cells. Because $C^{*}$ is in the subtree rooted at $C$, so is the cell $C^{\prime}$, and the theorem is proved for this case.

Note that the induction is valid because when Case 2 makes use of induction, the tree $C^{*}$ has fewer nodes. Thus, the process eventually terminates in Case 1 .

Figure 3 provides an illustration of Theorem 3.4.

\section{COMPLETE PROJECTION}

Given NuCAD $C$ and level $k$ on which to project, EasyProjection $(C, k)$ may fail to reduce the split-levels of all cells in $C$ to $k$ or below. If so, Theorem 3.4 tells us that there is a cell $C$ such that two children of $C$ with maximum split-level both have subtrees that contain a mix of TRUE and FALSE cells, and the split-level of these two child cells is greater than $k$, but the levels of the subtrees rooted at these two cells are less than or equal to $k$. So, to develop a complete projection algorithm, we need to be able to resolve exactly this situation. Algorithm 3, ProjectionResolve, does this. It takes two cells of split-level greater than $k$, both of which have subtrees of level at most $k$, and both of which have identical projections onto $k$-space. In our context, both cells will have the same split-level, though the algorithm is phrased more generally. It then refines the 


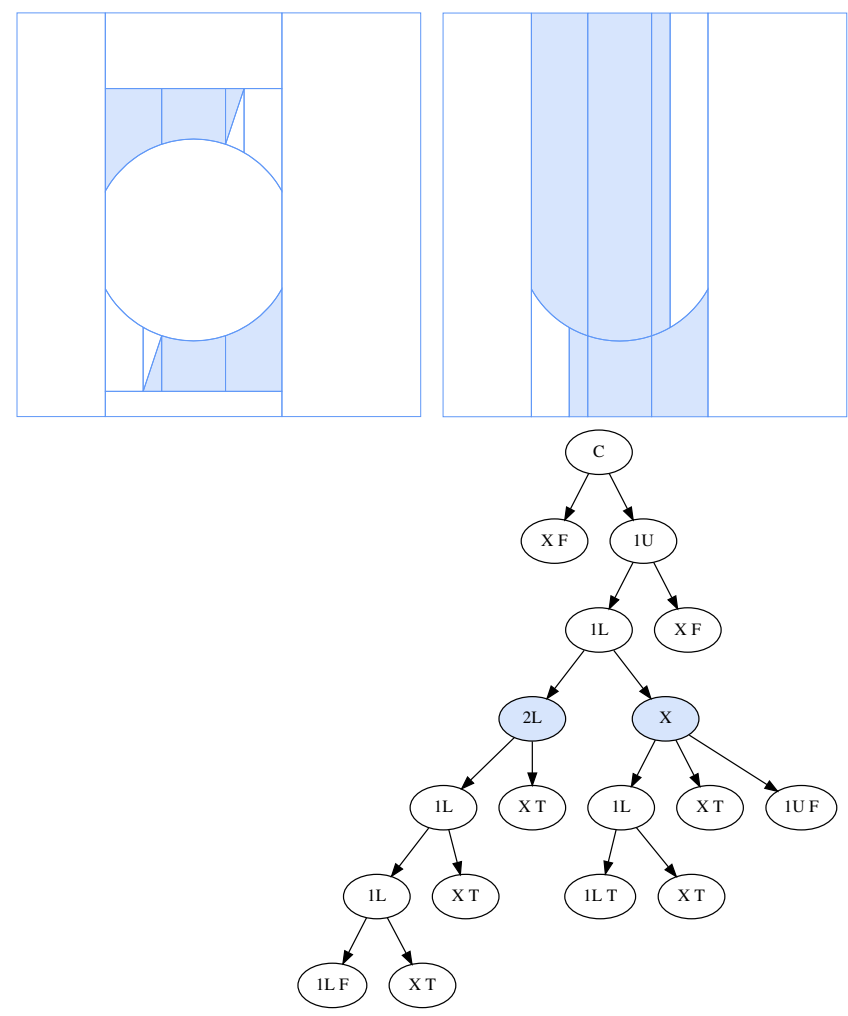

Figure 3: On the left is the NuCAD produced from the formula $x^{2}+y^{2}>1 \wedge y(y-3 x)>0 \wedge y<\frac{3}{2} \wedge y>-\frac{3}{2} \wedge x<\frac{7}{8} \wedge x>-\frac{7}{8}$. On the right is the NuCAD resulting from calling EasyProjection with $k=1$, and beneath it is the tree for that $\mathrm{Nu}$ CAD. Note EasyProjection did not succeed in eliminating all cells of split-level greater than $k$. The cell $C^{\prime}$ guaranteed by Theorem 3.4 is cell C1U1L and, as the two highlighted child cells have subtrees that, as promised, are of level lass than or equal to $k$, and contain both true and false cells.

subtree rooted at the first cell so that projection of its TRUE cells is (modulo a missing lower-dimensional component) equal to the union of the projections of the TRUE cells of both inputs. The second cell can afterwords be truncated to a leaf with truth value FALSE, and the projection onto $k$-space of all TRUE cells in the NuCAD will remain unchanged (modulo that missing lower-dimensional component).

Theorem 4.1. Algorithm 3 meets its specification.

Proof. We provide a proof of this theorem without covering cases in which locateLeaf or OCMerge return a FAIL result. Those FAIL results occur because we are dealing only with "open" NuCAD, which means situations in which sample points fall on the zero sets of certain polynomials have to be addressed by perturbing those sample points to move them off those zero sets. In [1], this is handled completely and rigorously proved correct. In this paper we handle it incompletely in the algorithm and ignore it in the correctness proof because we don't have the space to describe the perturbation completely, or cover its correctness. However, the perturbation process and the arguments of its correctness are essentially the same here as in [1].

Let $S$ be the union of the cells marked TRUE in $T_{1}$ and $T_{2}$, the subtrees rooted at $C_{1}$ and $C_{2}$ respectively, prior to the start of the algorithm. The essential invariant of this process is that, at the point of evaluating the continuation condition at line 2 , the union of the projections onto $k$ space of the cells in $Q$ and the cells marked TRUE in the subtree rooted at $C_{1}$ is a weak projection of $S$. In particular, it is equal to $S-Z$, where $Z$ is a subset of a variety defined by a non-zero polynomial in $x_{1}, \ldots, x_{k}$.

The proposed invariant condition is certainly true when we encounter the loop for the first time, as $C_{1}$ hasn't changed, and $Q$ contains exactly the TRUE cells from $T_{2}$, the subtree rooted at $C_{2}$ at the beginning of the algorithm. So we will show that if the condition is true at the beginning of a loop iteration, it is true at the end of that iteration.

Line 3 removes $c$ from $Q$, and line 4 sets $\alpha$ to the projection of $c$ 's sample point onto $r$-space. Line 5 locates a leaf node in the subtree rooted at $C_{1}$ that contains a point in $\alpha \times \mathbb{R}^{n-r}$. As explained above, we will assume here that this step is successful, and move to Step 11. At this point, $\pi_{r}(c) \cap \pi_{r}(d)$ is an open subset containing $\alpha$. OC-Merge is called on line 12 to compute $e$, a cylindrical cell in $r$-space that contains $\alpha$ and is contained within $\pi_{r}(c) \cap \pi_{r}(d)$. Once again, as explained above, we will assume that this operation does not fail.

At line 17 we make the call SplitByCell $(c, e, k)$. This will refine cell $c$ (i.e. it will no longer be a leaf node in $C_{2}$ ) so that its $X$ child's projection onto $r$-space is exactly $e$. The other child cells are enqueued on $Q$ so that the net change to the union of the cells on $Q$ is that one open set has been removed (corresponding to the $X$ child) and some points appearing on the boundaries may have been lost (but these are a subset of the variety defined by the product of all the polynomials defining boundaries.

If $d$ is marked TRUE, we are actually done, because the open set that was lost (the $X$-child) had a projection (cell $e$ ) that was a subset of the projection of $d$ (which is a TRUE cell in $C_{1}$ ) anyway. If $d$ is marked FALSE, then the algorithm splits $d$ by cell $e$. The new $X$-child is marked TRUE, so $e$ is picked up in the projection of $C_{1}$, and since the other new children of $d$ are marked FALSE, the projection of the leaves of $C_{1}$ is otherwise unchanged. Thus the condition holds.

Finally, we describe Algorithm 4, OpenNuCADProject. The correctness of OpenNuCADProject follows from previous theorems quite easily. Essentially, the only important observation is that, because ProjectionResolve $\left(C_{1}, C_{2}, k\right)$ sets all leaves in the subtree rooted at $C_{2}$ as FALSE, the following call to EasyProjection is guaranteed to merge two cells, and thus reduce the total number of cells in $C$ of split-level greater than $k$.

\section{EXPERIMENTAL RESULTS}

This section describes the results of experiments ${ }^{2}$ performed with a prototype implementation of Open NuCAD construction and the projection algorithm described here. These are available in TARSKI, a system for computing with Tarski formulas and semi-algebraic

${ }^{2}$ All experiments were performed on an Intel(R) Core(TM) i7-3770 CPU $3.40 \mathrm{GHz}$ with 8GB RAM. 

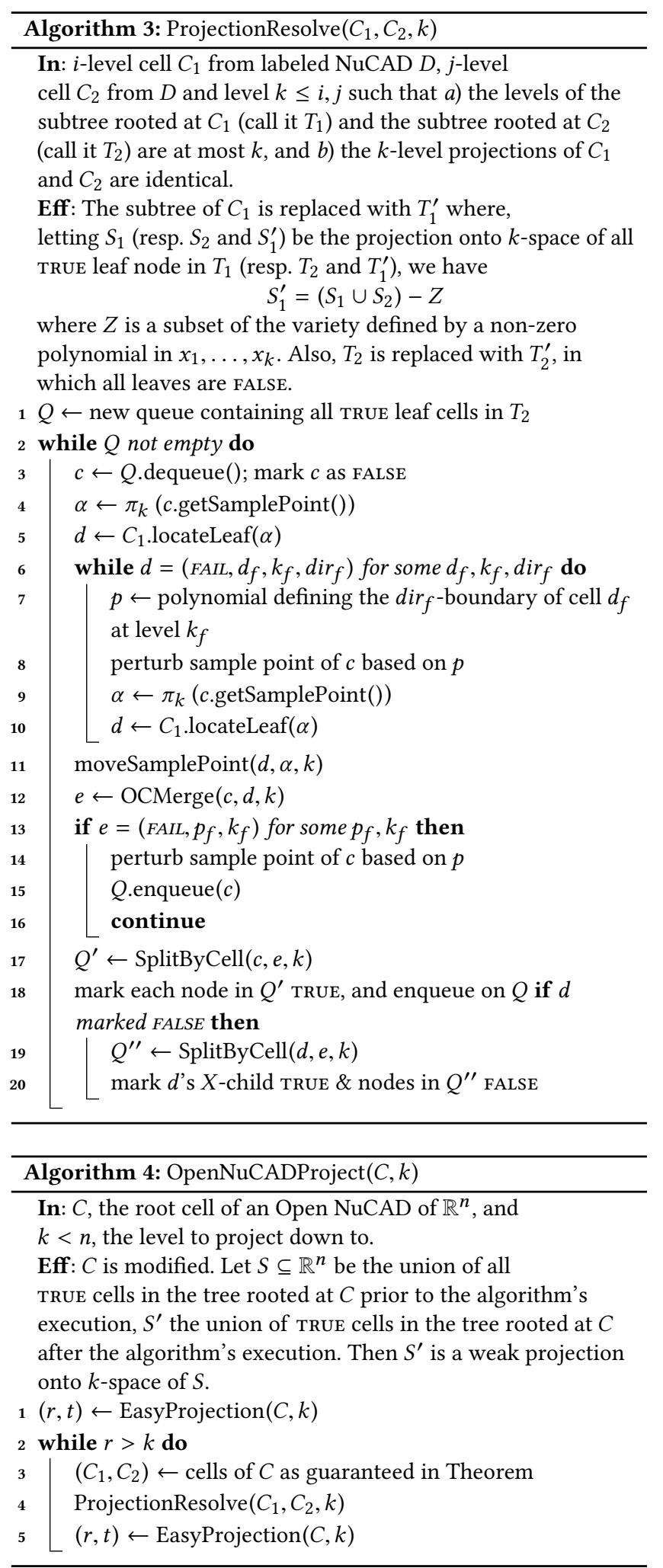

\begin{tabular}{|r|r|r|r|r|r|}
\hline & \multicolumn{2}{|c|}{$n=3$ variables } & \multicolumn{3}{|c|}{$n=4$ variables } \\
\hline$m$ & $r=1$ & $r=2$ & $r=1$ & $r=2$ & $r=3$ \\
\hline 4 & $5.2 \%$ & $4.1 \%$ & $5.8 \%$ & $10.0 \%$ & $7.6 \%$ \\
5 & $5.4 \%$ & $5.1 \%$ & $4.5 \%$ & $12.6 \%$ & $6.2 \%$ \\
6 & $5.0 \%$ & $5.9 \%$ & $3.8 \%$ & $16.6 \%$ & $11.7 \%$ \\
\hline
\end{tabular}

Figure 4: Time taken to compute the projection onto $r$-space of an Open NuCAD of $n$-space defined by $m$ inequalities, shown as a percentage of the time taken to compute the Open NuCAD of $n$-space in the first place. Percentage shown is the average of the 25 examples for each $(m, n, r)$ triple.

sets $^{3}$. Where referred to, Open CAD experiments are done with QEPCAD B ${ }^{4}$. The experiments used the "nonlinear set" of formulas ${ }^{5}$ from [1], which includes 25 formulas for each combination of $m$ inequalities on $n$ variables, where $m \in\{4,5,6\}$ and $n \in\{2,3,4\}$. Each inequality is randomly generated, degree two, and strict. There is good reason to be concerned about the meaning of experiments on "random" inputs like these, since the performance of many algorithms for computing with real polynomials behave differently (usually much worse!) on random input than input from actual applications. However, in this context it seems reasonable to do, since we are primarily interested in understanding how the $\mathrm{Nu}-$ $\mathrm{CAD}$ projection algorithm behaves as the parameters of number of variables, dimension to project onto and number of polynomials increase.

The first set of experiments investigates how the time required to compute the projection compares to the time required to compute the original NuCAD. The results, shown in Figure 4, show that the cost of computing the projection is generally small compared to the cost of computing the original Open NuCAD. ${ }^{6}$

The next set of data explores how the number of cells in the projected NuCAD compares with the number of cells in the original. It is not clear a priori that the number of cells will decrease, since the projection process requires subdividing cells. However, the results (shown in Figure 5) demonstrate that this is indeed the case, at least for the kinds of inputs considered here. ${ }^{7}$

We next compare computing an Open NuCAD for the projection of a semi-algebraic set given by a Tarski formula, to computing an Open $\mathrm{CAD}$ for the formula. Open CAD computations for projections were done with QEPCAD B, using settings that force it to do purely "Open" CAD constructions, in both free and bound variable space. It uses "partial" CAD, allowing it to terminate stack constructions in bound variable space early when a satisfying cell is found. Both TARSKI and QEPCAD B are built on top of the SACLIB computer algebra system, which requires deciding up-front on the size of the garbage collected array (GCA). For TARSKI, all computations

\footnotetext{
${ }^{3}$ www. usna. edu/Users/cs/wcbrown/tarski

${ }^{4}$ www. usna. edu/CS/qepcadweb/B/QEPCAD.html

${ }^{5}$ www. usna. edu/Users/cs/wcbrown/research/ISSAC17

${ }^{6}$ There was only one instance in all the tests for which the time for both was measurable (i.e. more than the $4 \mathrm{~ms}$ resolution of the timer) that the time for projection exceeded the time to construct: that was a $m=6, n=4, r=3$ example for which projection took $183 \%$ of the construction time. This example is labeled F15 in the data-set available at the URL given in the prior footnote.

${ }^{7}$ The worst example, in which the number of cells in the projected NuCAD is $70.3 \%$ of that in the original, came on example $m=6, n=4, r=3, \mathrm{~F} 15$ - the only example for which computing the projection actually took longer than computing the original NuCAD.
} 


\begin{tabular}{|r|r|r|r|r|r|r|}
\hline & $n=2$ & \multicolumn{2}{|c|}{$n=3$} & \multicolumn{3}{c|}{$n=4$} \\
\hline$m$ & $r=1$ & $r=1$ & $r=2$ & $r=1$ & $r=2$ & $r=3$ \\
\hline 4 & $32.3 \%$ & $4.9 \%$ & $32.9 \%$ & $0.3 \%$ & $5.5 \%$ & $35.6 \%$ \\
5 & $25.0 \%$ & $6.0 \%$ & $30.7 \%$ & $0.3 \%$ & $3.9 \%$ & $33.2 \%$ \\
6 & $24.7 \%$ & $6.7 \%$ & $28.6 \%$ & $0.1 \%$ & $3.9 \%$ & $32.7 \%$ \\
\hline
\end{tabular}

Figure 5: Number of cells in the projected NuCAD of $r$-space as a percentage of the number of cells in the original Open NuCAD of $n$-space. The percentage shown is the average of the 25 examples for each $(m, n, r)$ triple.

\begin{tabular}{|r|r|r|r|r|r|r|r|}
\hline \multicolumn{2}{|c|}{} & \multicolumn{2}{|c|}{ NuCAD } & \multicolumn{2}{c|}{ CAD } & \#cells \\
$m$ & $n$ & $r$ & \#cells & ex & \#cells & ex. & ratio \\
\hline 4 & 2 & 1 & 2.72 & 25 & 10.98 & 25 & 0.24772 \\
5 & 2 & 1 & 3.04 & 25 & 17.78 & 25 & 0.17098 \\
6 & 2 & 1 & 3.04 & 25 & 24.86 & 25 & 0.12228 \\
4 & 3 & 1 & 4.32 & 25 & 34.50 & 25 & 0.12522 \\
4 & 3 & 2 & 34.76 & 25 & 373.10 & 25 & 0.09317 \\
5 & 3 & 1 & 5.84 & 25 & 83.06 & 25 & 0.07031 \\
5 & 3 & 2 & 42.80 & 25 & 1342.94 & 25 & 0.03187 \\
6 & 3 & 1 & 5.40 & 25 & 189.46 & 25 & 0.02850 \\
6 & 3 & 2 & 41.16 & 25 & 4201.14 & 25 & 0.00980 \\
4 & 4 & 1 & 1.40 & 25 & 223.22 & 25 & 0.00627 \\
4 & 4 & 2 & 72.72 & 25 & 7618.18 & 25 & 0.00955 \\
4 & 4 & 3 & 826.88 & 25 & 86301.30 & 25 & 0.00958 \\
5 & 4 & 1 & 3.56 & 25 & 1943.50 & 25 & 0.00183 \\
5 & 4 & 2 & 128.12 & 25 & 131456.68 & 25 & 0.00097 \\
5 & 4 & 3 & 1492.20 & 25 & 1259602.00 & 23 & 0.00118 \\
6 & 4 & 1 & 4.44 & 25 & 13689.17 & 24 & 0.00032 \\
6 & 4 & 2 & 322.32 & 25 & 1023992.00 & 8 & 0.00031 \\
6 & 4 & 3 & 4108.88 & 25 & N/A & 0 & N/A \\
\hline
\end{tabular}

Figure 6: Number of cells in the projected Open NuCAD of $r$-space compared to the number of cells in the Open CAD of $r$-space. Number shown is the average of the completed examples for each $(m, n, r)$ triple. The "ex" columns shows how many of the 25 example computations actually ran to completion. Uncompleted example computations were not included in computing the cell count averages.

were done with a 10 million cell GCA. For QEPCAD B, 10 million was used for $n=2,100$ million for $n=3$ and 1 billion for $n=4$. Still, a number of QEPCAD Bcomputations failed for lack of memory. Figure 6 compares the number of cells in the open decompositions of $r$-space ultimately produced. Figure 7 compares the time required on the various 4-variable problem sets. Because QEPCAD B, using partial CAD, does not have to construct a decomposition of $n$ space, it is at an advantage for $r=1$. (Development of a "partial" version of $\mathrm{NuCAD}$ is an avenue for future research.) However, as $m$ and $r$ increase, the complexity of the original semi-algebraic set and its projection increase, and Open NuCAD becomes faster by several orders of magnitude, despite naive and unoptimized implementation in TARSKI and the fact that QEPCAD B uses partial CAD, and TARSKI has to compute a full Open NuCAD of $\mathbb{R}^{n}$.

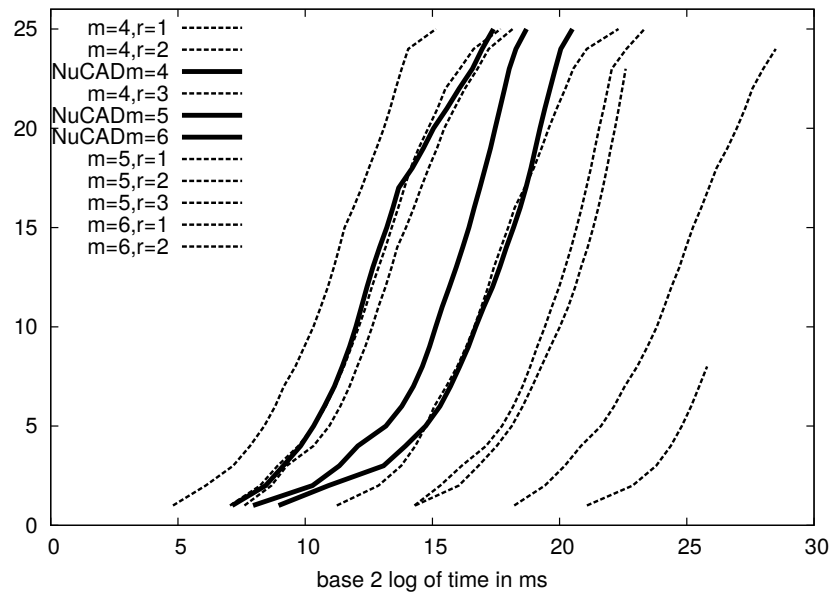

Figure 7: This plot shows number of examples (of the 25 per data set) completed as a function of time spent, where examples are ordered from least to greatest execution time. Only 4-variable examples are considered. The dotted lines represent Open partial-CAD computations for different $m$ and $r$ values, where $r$ is the dimension projected onto and $m$ is the number of inequalities. The solid lines show computations for Open NuCADs of 4-space (since previous results show the time to project is typically small relative to the time required to construct the NuCAD). Solid lines show times to compute complete Open NuCADs of 4-space, time to then project is left out to improve readability.

\section{CONCLUSION}

This paper provided an algorithm for computing (weak) projections of semi-algebraic sets in the (Open) NuCAD representation. The experiments reported here indicate the potential of this approach. However, there is still much work to be done, including extending the theory of NuCAD beyond the "open" case, and along with that, extending the projection algorithm to general NuCADs.

This work was supported by National Science Foundation grant number 1525896. The author would like to thank the anonymous referees for their insightful comments, and also to express his hope that his revisions addressed their concerns.

\section{REFERENCES}

[1] Christopher W. Brown. Open non-uniform cylindrical algebraic decompositions. In Proceedings of the 2015 ACM on International Symposium on Symbolic and Algebraic Computation, ISSAC '15, pages 85-92, New York, NY, USA, 2015. ACM.

[2] Christopher W. Brown and James H. Davenport. The complexity of quantifier elimination and cylindrical algebraic decomposition. In ISSAC '07: Proceedings of the 2007 international symposium on Symbolic and algebraic computation, pages 54-60, New York, NY, USA, 2007. ACM.

[3] Dejan Jovanović and Leonardo de Moura. Solving Non-linear Arithmetic. In Bernhard Gramlich, Dale Miller, and Uli Sattler, editors, Automated Reasoning, volume 7364 of Lecture Notes in Computer Science, pages 339-354. Springer Berlin Heidelberg, 2012.

[4] S. McCallum. Solving polynomial strict inequalities using cylindrical algebraic decomposition. The Computer fournal, 36(5):432-438, 1993

[5] A. Strzebonski. Solving systems of strict polynomial inequalities. Journal of Symbolic Computation, 29:471-480, 2000.

[6] Adam Strzeboński. Cylindrical algebraic decomposition using local projections. ISSAC '14, pages 389-396, New York, NY, USA, 2014. ACM. 